\title{
The manufacture and properties of oil palm and pineapple leaf fiberboard panels
}

\author{
R. Moya ${ }^{1}$, D. Camacho ${ }^{1}$, J. Mata $^{2} \&$ R. Soto Fallas ${ }^{3}$ \\ ${ }^{1}$ Escuela de Ingeniería Forestal, Instituto Tecnológico de Costa Rica, \\ Costa Rica \\ ${ }^{2}$ Escuela de Química, Universidad de Costa Rica, Costa Rica \\ ${ }^{3}$ Escuela de Química, Universidad Nacional, Costa Rica
}

\begin{abstract}
Particleboards were manufactured from oil palm fruit, the oil palm mesocarp fiber of Elaeis guineensis, the leaves of pineapple (Ananas comosus) and the sawdust from three fast-growing species of trees (Gmelina arborea, Tectona grandis and Cupressus lusitanica). The chemical and anatomical compositions of E. guineensis and A. comosus and their effects on urea-formaldehyde adhesive were investigated. Afterwards, the particle combination of the fiber of E. guineensis and A. comosus with the sawdust of three species was investigated. The results showed that the fiber of Elaeis guineensis has a higher oil content than that of the pineapple leaf, and the pineapple leaf has large fiber of over $5 \mathrm{~mm}$. The fiber of E. guineensis must be washed with water to increase the adhesion of particles. The best combination of agricultural fiber and sawdust of fast growth tropical species is $50 \%-50 \%$, respectively (waste agriculture and sawdust, w/w\%). It was shown that the pineapple leaf and the empty fruit of oil palm can be substituted for conventional wood-based particleboards.

Keywords: tropical species, lignocellulosic wastes, Tetra Pak.
\end{abstract}

\section{Introduction}

Tropical regions have environmental factors that favor excellent levels of productivity of agricultural crops [1]. Currently, it is estimated that 47,000 hectares of oil palm and 40,000 hectares of pineapple are planted in Costa Rica [2]. Unfortunately, the residues generated by this agricultural activity 
have not been used appropriately and have generated a significant level of contamination [3]. Khalil et al. [4] mention that an oil palm plantation produces about 350 tons of residues/ha/rotation while Ulloa et al. [3] found that a pineapple plantation produces around 220 tons of residues/ha/rotation. These residues contain basically lignocellulosic components and their limited use has been attributed primarily to a lack of technology for their processing and secondly to the specific application of potential products [5].

On the other hand, the variety of packages launched by Tetra Pak (a multinational food packaging and processing company of Swedish origin) generates a large amount of waste residues worldwide. It is reported that around 150 trillion of these packages were produced in 2010 (www.tetrapak.com) for milk, juice, nectars and others [6], which were converted in residues after their consumption. These products decompose slowly and high technology such as plasma treatment is required to recycle them [7].

The feasibility of using woody biomass in combination with agricultural wastes and Tetra Pak residues in particleboard fabrication presents an encouraging challenge and corresponds to the main goal of our current research. It has been reported that approximately 81.5 million $\mathrm{m}^{3}$ of particleboards were produced in 2004 and their production continues to grow [8].

The objective of this study was to evaluate the physical, mechanical and physical properties of particleboards prepared with woody biomass in combination with agricultural residues and separately with discarded Tetra Pak packages. Woody biomass corresponded to three species planted for commercial purposes in tropical areas (Cupressus lusitanica, Gmelina arborea and Tectona grandis); agricultural wastes corresponded to pineapple leaves (from the crown and the plant) and oil palm (fruit and bunch); Tetra Pak packages were residues obtained from recycling centers located at Cartago downtown in Costa Rica.

\section{Material and methods}

\subsection{Raw Materials}

The woody biomass corresponds to Gmelina arborea (GA) from a 9 year old plantation, Tectona grandis (TG) from a 16 year old plantation, and Cupressus lusitanica (CL) from a 22 year old plantation (all of them used for commercial reforestation in tropical countries); Agriculture wastes consisted of pineapple leaves (PL) and residues of oil palm from the extraction of oil empty fruit bunch of oil palm (EBF) and oil palm mesocarp fiber of the fruit (OPMF). PL came from an 18 month old plantation and they were used from the crown (PLC) and from the plant (PLP); and Tetra Pak packages residues (TP) were obtained from recycling centers located at Cartago downtown in Costa Rica.

\subsection{Material preparation}

Pineapple leaves and oil palm residues were dried following the methodology given by Tenorio and Moya [8]. OPMF residues were washed for one hour in hot water stirring continuously, in order to obtain the best performance with 
adhesives. TP were washed to eliminate residual contents and then they were cut into $1 \mathrm{~cm}$ wide strips, using a paper cutter. The three woody species were chipped to size less than $3 \mathrm{~mm}$. Then a Retsch cutting mill was used to reduce the dried chips into particles that resulted of sizes between 0.7 and $6.0 \mathrm{~mm}$. Finally, the particles of each material were placed into a climate-controlled chamber to obtain $6 \%$ equilibrium moisture content.

\subsection{Particleboard preparation}

Blends of woody biomass and residues (agricultural and TP packages) used for the particleboard preparation are presented in Table 2 . They all were prepared using a 50:50 ratio. The adhesive used was urea-formaldehyde (UF) with $62 \%$ solids, and the adhesive application corresponded to percentages $8 \%$ with respect to the total weight of the particleboard. The amount of adhesive applied was taken from previous research [5]. In total, fifteen different blends were prepared and twenty $35 \times 35 \mathrm{~cm}$ boards were obtained from each mixture. The target particleboard density was of $0.65 \mathrm{~g} / \mathrm{cm}^{3}$, with an average thickness of $12.5 \mathrm{~mm}$ and 3 layers. The two external layers or faces $(2 \mathrm{~mm}$ thick $)$ contained fine particles 0.7 to $1.5 \mathrm{~mm}$ long while the inner layer (core) contained thicker particles 1.5 to $6.0 \mathrm{~mm}$ long. Particleboards were pressed at $25 \mathrm{MPa}$ and $175^{\circ} \mathrm{C}$ for 10 minutes and after that they were put into a climate-controlled chamber during 24 hours to homogenize their moisture content and to finish their adhesive curing process.

\subsection{General properties determination}

Particleboard specimens of $2.5 \mathrm{~cm}$ (width) x $2.5 \mathrm{~cm}$ (length) $\times 1.2 \mathrm{~cm}$ (thickness) were cut from each particleboard to measure their thickness, rugosity, and color. The surface of each particleboard was not sanded nor did it receive any preparation. Thickness was measured using a micrometer caliper in four places of the specimens and the average of the measurements was determined; Rugosity was measured in two different points of the specimens using a Starrett rugosity meter (Model No.3800). Color of the specimens was determined using a Hunter Lab mini Scan XE Plus spectrophotometer. The range of this measurement was from 400 to $700 \mathrm{~nm}$ with an opening at the point of measurement of $11 \mathrm{~mm}$. For the observation of reflection, the specular component (SCI mode) was set at a $10^{\circ}$ angle, which is normal for the specimen surface (D65/10); a field of vision of $2^{\circ}$ (Standard observer, CIE 1931) and an illumination standard of D65 (corresponding to daylight in $6500 \mathrm{~K}$ ). The mini Scan XE Plus generated three parameters $\left(\mathrm{L}^{*}, \mathrm{a}^{*}\right.$ and $\left.\mathrm{b}^{*}\right)$ for each measurement using CIEL*a*b* colour system. Coordinate $\mathrm{L}^{*}$ stands for lightness and represents the position on the black-white axis $\left(\mathrm{L}^{*}=0\right.$ for black, $\mathrm{L}^{*}=100$ for white); coordinate $\mathrm{a}^{*}$ for the position on the red-green axis (positive values for red, negative values for green); and coordinate $\mathrm{b}^{*}$ for the position on the yellow-blue axis (positive values for yellow, negative values for blue) [10]. 


\subsection{Physical and mechanical properties}

Moisture content (MC) was evaluated according to the ASTM D-4442 standard [11]. Density, dimensional stability measured by dimensional change, swelling (SW) and water absorption (WA) were determined according to the ASTM D-1037 standard [12] (ASTM 2012a). Nine mechanical tests were conducted on the particleboards fabricated. They corresponded to: static bending [to get the Module of rupture (MOR) and the Modulus of elasticity (MOE)]; tensile strength parallel to surface (T//S); tensile strength perpendicular to surface $(\mathrm{T} \perp \mathrm{S})$; hardness $(\mathrm{HR})$; glue-line shear $(\mathrm{GLS})$; shear in the plane of the panel (SPS); nail withdrawal (NWI); nail-head pull-through (NHP); and direct screw withdrawal (DSW). The ASTM D-1037 standard was used in all mechanical properties determinations [11].

\subsection{Statistical analysis}

The statistical analysis of the general, physical and mechanical properties was performed using the one-way ANOVA test to find out significant statistical differences between properties of the particleboards prepared. For those properties that showed significant differences a Tukey test was applied with a significance level of $\mathrm{P}<0.05$. For all these analyses, the SAS 8.1 statistics program for Windows (SAS Institute Inc., Cary, N.C.) was used. In addition, a hierarchical cluster multivariate analysis was applied on all the variables measured to find the degree of similarity of the different types of particleboards fabricated. The average value of each property was introduced in the model

\section{Results and discussion}

\subsection{General properties}

Thickness, rugosity, color, density and moisture content of the particleboards fabricated are presented in Table 1. Although the target thickness was $12.5 \mathrm{~mm}$, a variation between 12.0 to $13.5 \mathrm{~mm}$ was obtained. No significant differences in thickness were found in the particleboards manufactured with GA and TG woody biomass; however, with CL there were statistical differences between some of its blends. Blends composed by both CL and OPMF and CL and EFB presented statistically higher thickness values than those of the remaining $\mathrm{CL}$ mixtures, which did not present significant statistical differences.

The evaluation of rugosity of the particleboards showed a variation between 9.1 to $12.0 \mu \mathrm{m}$ (Table 1). For CL it was found that particleboard prepared with pineapple leaves from the crown (CL-PLC) and oil palm mesorcarp fiber (CLOPMF) presented statistically higher values of rugosity than those of the other three mixtures of CL.

Meanwhile, for GA differences of rugosity were only found in particleboard prepared with Tetra Pak (GA-TP) and pineapple leaves from the plant (GAPLP), which was statistically lower than the other three blends. Lastly, no differences between the values of rugosity were found for the five blends of TG. 


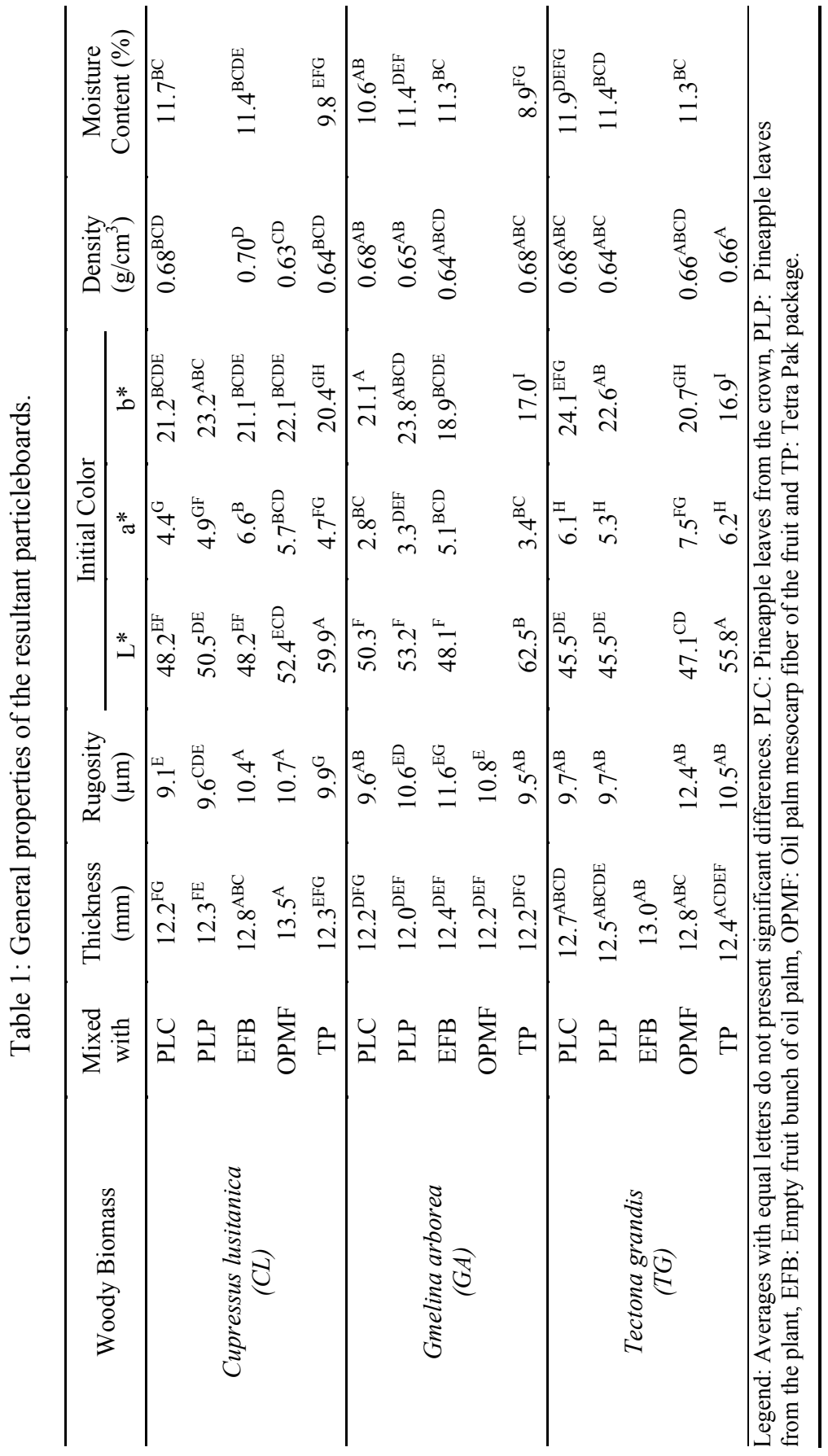


In the evaluation of color, the values of $\mathrm{L}^{*}$ (luminosity), $\mathrm{a}^{*}$ (redness) and $\mathrm{b}^{*}$ (yellowness) were higher than zero (Table 1). The $\mathrm{L}^{*}$ parameter was statistically higher for the mixtures of the three woody biomass species in combination with $\mathrm{TP}$, while the remaining four blends of each species did not present significant differences. Regarding the $\mathrm{a}^{*}$ parameter, for CL particleboards it was found that the mixtures with pineapple (PLP and PLC) presented statistically higher values compared to the mixtures with oil palm and TP. In the case of GA particleboards, the mixtures with oil palm (EFB and OPMF) presented the statistically highest values. Meanwhile, for TG particleboards, the $\mathrm{a}^{*}$ value was significantly higher for particleboards with the OPMF. Lastly, for the values of $b^{*}$ parameter in CL particleboards, significant differences were only found in the CL-TP mixture, which was significantly lower than the remaining four mixtures. In contrast, GA and TG particleboards gave significantly higher values for $b^{*}$ in the mixtures containing pineapple leaves (PLC and PLP).

\subsection{Physical properties}

The resultant density for the particleboards varied from 0.62 to $0.70 \mathrm{~g} / \mathrm{cm}^{3}$ (Table 2). No significant differences in density were found for particleboards fabricated with the same woody biomass and agricultural or TP residues. In terms of $\mathrm{MC}$, the particleboards presented a variation between $9.1 \%$ to $11.9 \%$. All particleboards prepared with TP presented the lowest statistically significant moisture content values compared to the rest of the mixtures. Swelling (SW) in the particleboards varied from $20 \%$ to $90 \%$. Those panels fabricated with the three woody biomass species and TP presented statistically the lowest SW values (Fig. 1a). Particleboards prepared with the woody biomass GA in combination with PLC, EFB and OPMF presented the highest swelling values (Figure 1a).

Regarding the water absorption (WA) of particleboards, the results varied from $79.5 \%$ to $170.8 \%$. The WA behavior is similar to the SW behavior; that is, particleboards fabricated with TP presented the lowest values and the highest values corresponded to the particleboards fabricated with PLC. Dimensional stability measured by dimensional change $(\%)$ on the other hand varied from 0.3 to $0.7 \%$ in dimension (Figure $1 \mathrm{~b}$ ). The highest values of dimensional change for all particles resulted for woody biomass in combination with OPMF and the lowest dimensional change was presented in particleboards fabricated with TP (Figure 1c).

The results for the static bending test, that is MOE and MOR, are presented in Figure 2. CL and TG woody biomass mixed independently with both OPMF and TP demonstrated statistically superior values of MOE and MOR than the rest of the combinations (Figure $2 \mathrm{a}$ and $2 \mathrm{~b}$ ). The lower MOR and MOE values were found for woody biomass GA and TG in combination independently with pineapple leaves (PLC and PLP) and EFB (Fig. 2a and 2b).

As presented in Table 2, T//S test in particleboards with CL, the CL-OPMF mixture presented a significantly higher value compared to the other four mixtures, which did not differ from each other. In particleboards with GA, the GA-OPMF mixture was also the highest, while the mixtures with pineapple (PLP and PLC) presented significantly lowest values. 


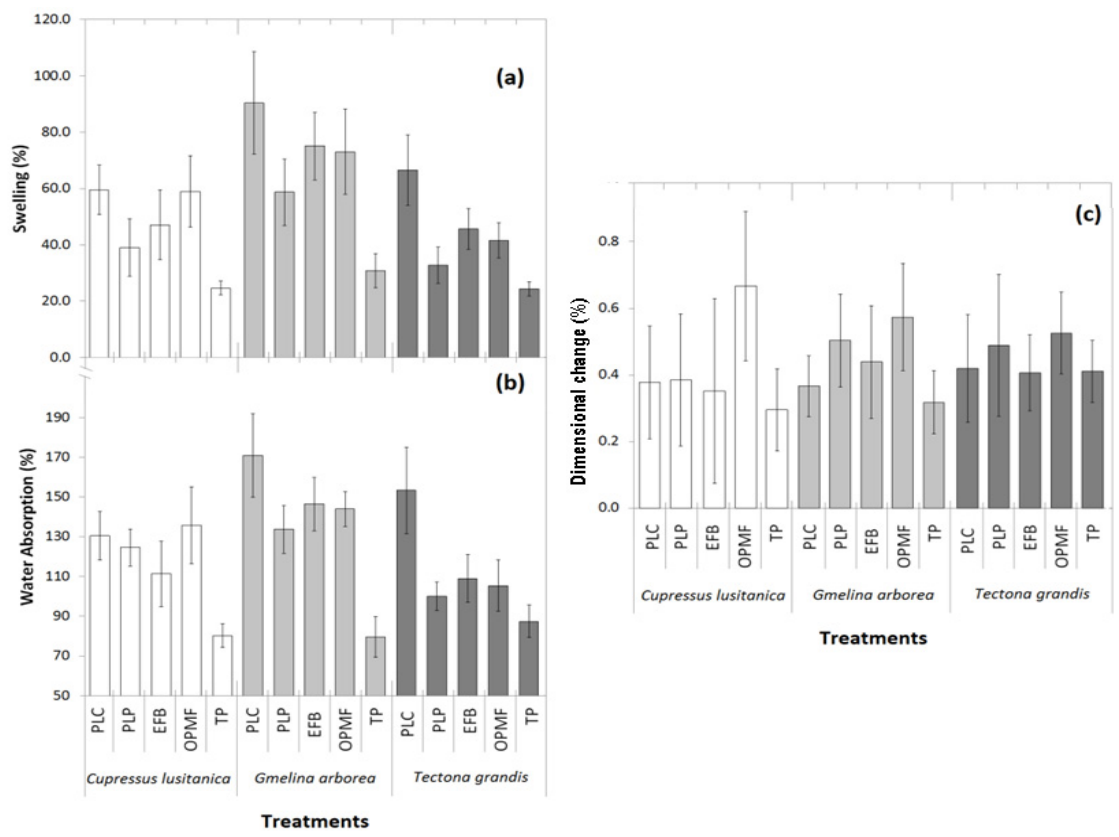

Figure 1: $\quad$ Physical properties in particleboards manufactured with Cupressus lusitanica, Gmelina arborea and Tectona grandis mixed with pineapple leaves, fiber from oil palm fruit and Tetra Pak packages, showing results of (a) swelling, (b) water absorption and (c) dimensional change.
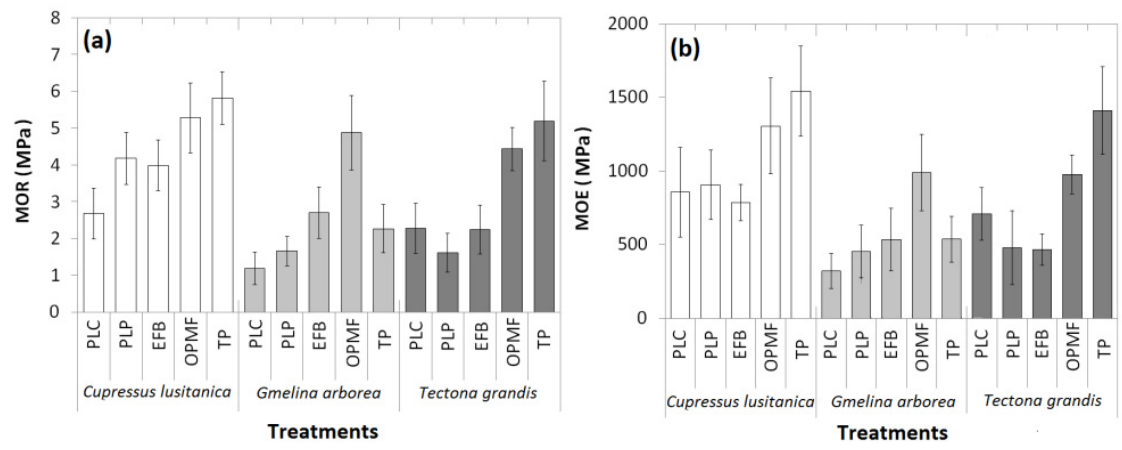

Figure 2: $\quad$ MOR (a) and MOE (b) in static bending test in particleboards of Cupressus lusitanica, Gmelina arborea and Tectona grandis mixed with pineapple leaves, fiber from oil palm fruit and Tetra Pak packages.

Finally, for TG, particleboards prepared with TG-OPMF and TG-TP resulted with significantly highest T//S values (Table 2). Regarding the HR, only the CL in combination with PLC presented a significantly low HR value compared with 
all other mixtures No significant differences were found in HR for particleboards prepared with GA. Finally, for particleboards prepared with TG, the mixtures TG-TP, TG-OPMF and TG-EFB mixtures presented significantly higher HR values than those of pineapple mixtures.

Regarding the resistance in $\mathrm{T} \perp \mathrm{S}$, in particleboards with $\mathrm{CL}$, the CL-TP mixture presented the highest value. For GA, the GA-TP and GA-OPMF mixtures presented higher values of $\mathrm{T} \perp \mathrm{S}$ compared to the remaining three mixtures (Table 2). The glue-line shear test (GLS) gave higher resistance for particleboards prepared with TP and two of the woody biomass species: CL and TG. The same trend was found SPS resistance, that is, the higher values were determined for CL and TG particleboards with TP (Table 2). Regarding the tests involving nails (NWI and NHP) (Table 2), for CL particleboards, the CL-PLC presented the lowest significant value for nail withdrawal (NWI). No significant differences were found between the mixtures for GA. In the case of TG particleboards, TG-OPMF and TG-TP mixtures had the highest significant values. For the NHP test in CL particleboards, only the CL-PLC mixture showed values significant lowest difference in relation with the rest of the mixtures. The GA particleboards in mixture with OPMF presented significantly higher nail resistance values compared with their remaining mixtures. Finally, TG-OPMF and TG-TP mixtures presented significantly higher values of resistance in NHP. TG-PLP mixtures presented the lowest significant resistance in NHP of the TG mixtures (Table 2).

In the DSW test, the particleboards with all three woody biomass types (CL, GA and TG) mixed with TP presented significantly higher resistance values compared to the mixtures with agricultural residues. The performance of these remaining four agricultural mixtures varied with the woody species used. For particleboards prepared with CL and PLC, the DSW was significantly lower compared to the reminding mixtures. GA and TG particleboards did not present significant differences in DSW values of the mixtures with pineapple leaves and oil palm components (Table 2).

\section{Conclusions}

Woody biomass CL displayed the best mechanical performance compared with GA and TG when it is combined in turn with agricultural wastes and with TP residues. CL presented the highest values in MOR and MOE in flexure, tensile strength parallel to surface, glue-line shear, SPS: shear in the plane of the panel, nail withdrawal, and nail-head pull-through.

Particleboards prepared with woody biomass (CL and TG) in combination with TP residues present improved properties in terms of their lower water absorption, higher dimensional stability and higher mechanical properties compared to the particleboards prepared with woody biomass and all agricultural wastes. The improvement ranged from $40 \%$ in most of the physical properties (water absorption, swelling, dimensional stability) up to $100 \%$ in most of the mechanical properties (bending, tensile, hardness, shear and nail tests). 


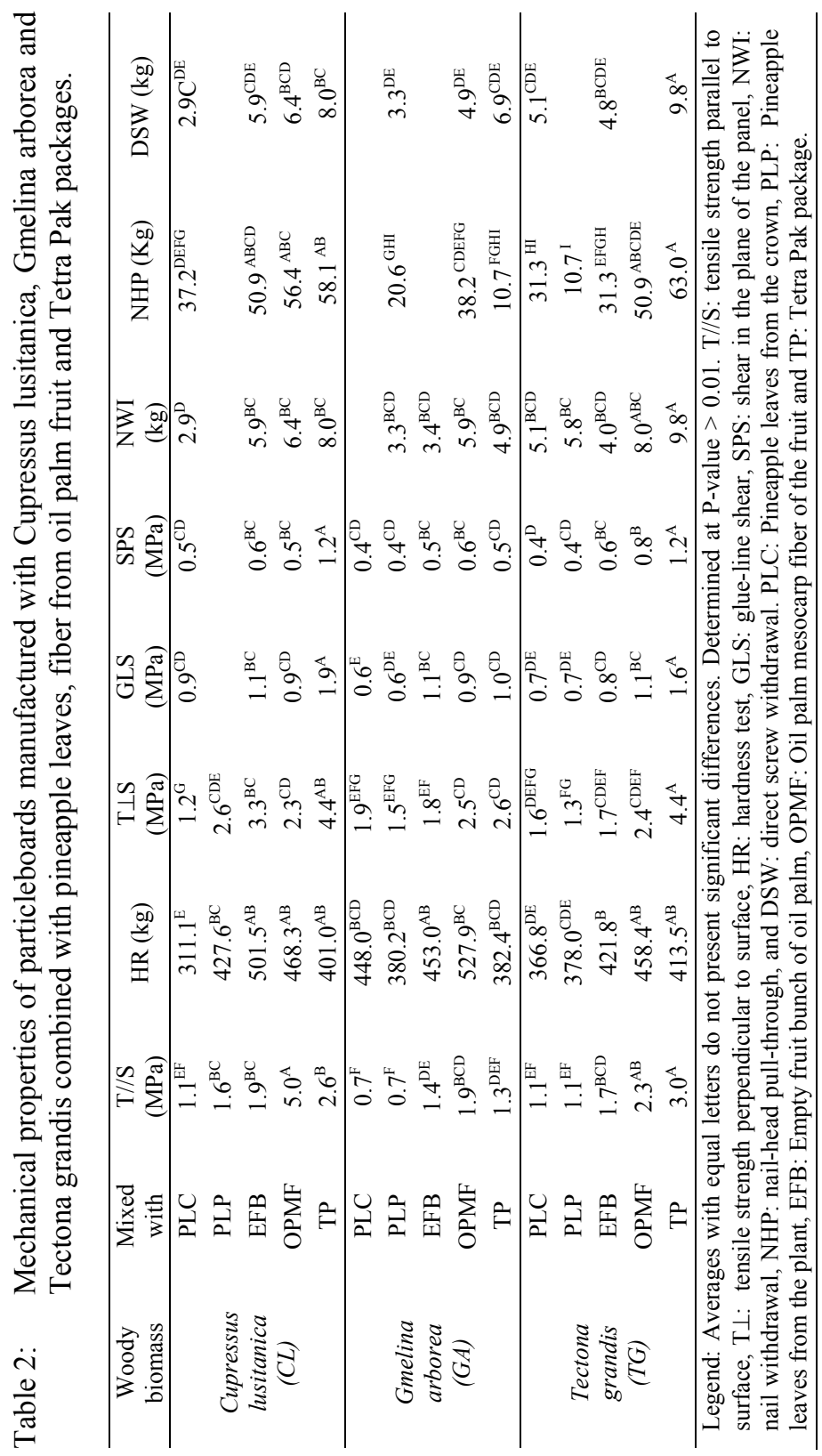


In terms of the particleboards prepared with all three woody biomass types and only agricultural wastes, the best performance was demonstrated for the agricultural waste of OPMF. Conversely, the woody biomass mixtures with pineapple residues presented the lowest performance in most of the mechanical tests.

Most of the particleboards prepared with woody biomass and agricultural wastes presented reddish and yellowish shades, typical of tropical wood. However, particleboards with woody biomass and TP produces color with high values of $\mathrm{L}^{*}$, giving a more white or clear coloration in the surface of the particleboards. This is a desirable property.

\section{Acknowledgements}

We thank the "Vicerrectoría de Investigación y Extensión" of the "Instituto Tecnológico de Costa Rica" and CONARE for their financial support. Also we give thanks to PINDECO and COOPEAGROPAL for providing the raw materials and facilities for this study.

\section{References}

[1] Bertsch, F. El recurso de la tierra en Costa Rica. Agronomía Costarricense, 30, 133-155, 2005.

[2] GFA Consulting Group. (2010) Informe Final: Estudio del Estado de la producción sostenible y propuesta de mecanismos permanentes de fomento de la producción sostenible. San José, Costa Rica.

[3] Ulloa, J.B., Weerd, J.H., Huisman, E.A. \& Verreth J.A. (2004) Tropical agricultural residues and their potential uses in fish feeds: the Costa Rica situation. Waste Management, 24, 87-97.

[4] Khalil, H. Ismail, A.H., Ahmad, M.N., Ariffin, A. \& Hassan, K. The effect of various anhydride modification on mechanical and water absorption properties of oil palm empty fruit bunches reinforced polyester composites, Polymer Industrial 50:1-9, 2001.

[5] Hidalgo, M. Manufacturing rigid board by packaging waste containing aluminum and polyethylene, Journal Science Industrial Research, 70, 232-234, 2011.

[6] Korkmaz, A., Yanik, J., Brebu, M. \& Vasile, C. Pyrolysis of the Tetra Pak. Waste Management, 29, 2835-2841, 2009.

[7] Saravia- Cortez, A.M., Herva, M., García-Diéguez, C. \& Roca, E. (2013) Assessing environmental sustainability of particleboard production process by ecological footprint. Journal of Cleaner Production, 52, 301-308.

[8] Tenorio, C. \& Moya, R. Evaluation of different methods proposal for the drying of lignocelluloses residues. BioResources, 7, 3500-3514. 2012.

[9] Hunterlab. The measurement of apparence. Published by John Wiley \& Sons, Inc. New York, USA. 1995. 
[10] ASTM D4442. Standard Test Methods for Direct Content Measurement of Wood and Wood-Base materials. ASTM International; Philadelphia, PA. USA, 2012a.

[11] ASTM D1037. Standard Test Methods for Evaluating Properties of WoodBase Fiber and Particle Panel Materials, ASTM International, Philadelphia, PA. USA, 2012b. 